\title{
Primary paratesticular osteosarcoma: A case report
}

\author{
RAN HONG $^{1}$, GEUNJAE LEE ${ }^{2}$, HYUNGSANG KIM ${ }^{2}$, CHUL-SUNG KIM $^{2}$ and KEUN-HONG KEE ${ }^{1}$ \\ Departments of ${ }^{1}$ Pathology and ${ }^{2}$ Urology, Medical School, Chosun University, Gwangju 504-140, Republic of Korea
}

Received September 19, 2011; Accepted November 24, 2011

DOI: $10.3892 / \mathrm{ol} .2011 .518$

\begin{abstract}
Extraosseous osteosarcoma originating from paratesticular soft tissue is an extremely rare type of malignant tumor. With the exception of the present study, only one other case of osteosarcoma originating from paratesticular soft tissue has previously been reported. A 52-year-old man presented with a painless scrotal swelling and palpable mass. The patient underwent left orchiectomy with the diagnosis of testicular tumor. Pathological examination was suggestive of osteosarcoma without any different histological components. The postoperative course of the patient was uneventful, and the patient is currently free of disease.
\end{abstract}

\section{Introduction}

Primary paratesticular sarcoma comprises a unique subset of intrascrotal sarcoma that is neither associated with germ cell nor testicular elements. Primary osteosarcoma of paratesticular soft tissue is a rare tumor, with few reports in the literature (1-3). To the best of our knowledge, only one case (4) arising directly from paratesticular soft tissue has been reported. Due to the rarity of paratesticular osteosarcoma, histogenesis, treatment and specific survival rates are not available. In this study, we report a second case of primary osteosarcoma of paratesticular soft tissue with a review of the literature.

\section{Case report}

A 52-year-old man presented with painless swelling and a palpable mass of the left scrotum, which showed a steady enlargement over the course of a year. Physical examination revealed a hard and non-tender mass of the left scrotum. Clinical impression was testicular tumor and a radiological evaluation was performed. A pelvic CT scan revealed a large paratesticular mass with calcification and internal necrosis in the left scrotum, which displaced the left testis inferiorly (Fig. 1). No enlargement of the lymph node was detected. Testicular ultrasonography also revealed a heterogeneous

Correspondence to: Dr Keun-Hong Kee, Department of Pathology, Chosun University Hospital, 588 Seosuk-dong, Dong-gu, 501-140 Gwangju, Republic of Korea

E-mail:nanih@chosun.ac.kr

Key words: extraosseous osteosarcoma, paratestis mass of the left scrotum. Radiological findings suggested a paratesticular sarcoma arising from the left spermatic cord. The patient underwent a left orchiectomy. The specimen was $14.0 \times 5.5 \times 5.0 \mathrm{~cm}$ in size, containing the left testis, spermatic cord and a gray-tan colored mass. Serial sectioning revealed a $6.5 \times 5.0 \mathrm{~cm}$-sized mass displacing the left testis inferiorly. The mass was well circumscribed by a thick fibrous capsule, solid and firm, and exhibited focal necrosis and hemorrhage (Fig. 2). The testis, epididymis and spermatic cord were intact. Microscopically, the tumor cells were highly cellular with spindle or polyhedral cells that were cytologically atypical and were mitotically active, frequently demonstrating atypical mitotic figures. Portions of osteoid and marked bony differentiation were frequently noted (Fig. 3). Immunohistochemically, the tumor cells were reactive for vimentin, but not for smooth muscle actin, CD34, cytokeratin, desmin, inhibin, myo-D1 and S-100 proteins. We diagnosed this tumor as an extraosseous osteosarcoma of paratesticular soft tissue. The postoperative course of the patient was uneventful, and the patient has remained disease-free for 9 months. The research protocol was approved by the Ethics Committee of Chosun University Hospital (IRB-11S-235).

\section{Discussion}

Paratesticular tumors may be clinically indistinguishable from testicular tumors, particularly when they are large. They usually present as a scrotal mass or swelling, with or without pain. In certain cases, they may be clinically misinterpreted as an inguinal hernia (4). Extraskeletal osteosarcoma is a malignant mesenchymal neoplasm that produces osteoid, bone or chondroid material, and is located in the soft tissue without attachment to the skeleton. Compared with osteosarcoma of the bone, extraskeletal osteosarcoma is rare, accounting for $1-2 \%$ of all soft tissue sarcomas (5-7). Genitourinary sarcomas are also rare in adults and are estimated to comprise less than $2.7 \%$ of all sarcomas. Russo et al reported that of the 43 cases of genitourinary sarcoma, the most common site of the tumor was paratesticular, followed by the prostate/seminal vesicle, urinary bladder and kidney, and that the most common histological type was leiomyosarcoma, followed by rhabdomyosarcoma and liposarcoma (8). Similarly, the paratestis is a relatively common site of sarcoma; however, osteosarcoma arising directly from paratesticular soft tissue is extremely rare. The first reported case was that of a 52-year-old man who presented with left scrotal swelling (4). Histological examination of the left orchiectomy specimen revealed a 


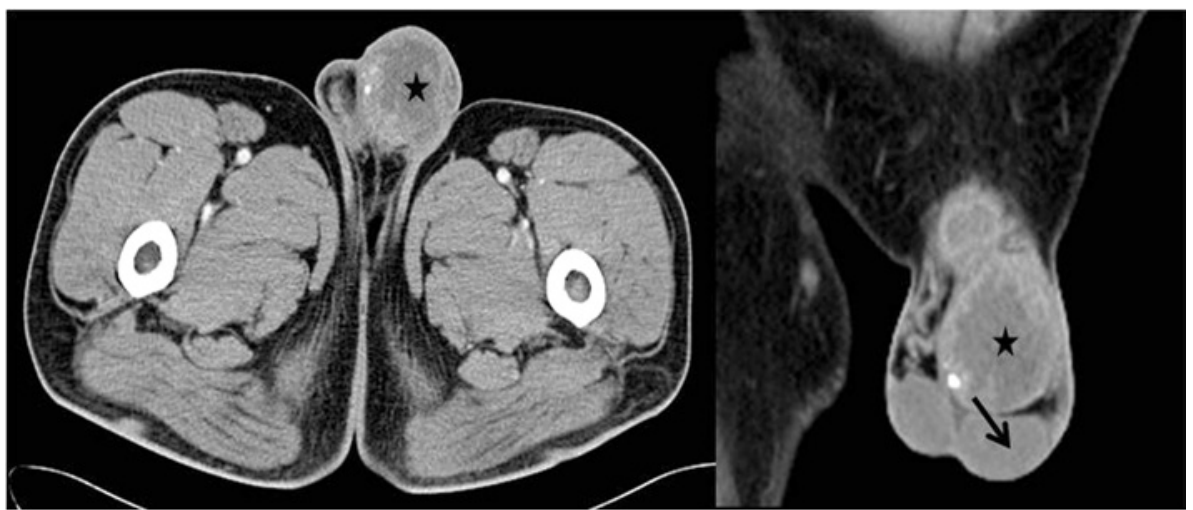

Figure 1. Computed tomography scan revealing a 10x12 cm-sized left paratesticular mass (star) displacing the left testis (arrow) inferiorly.

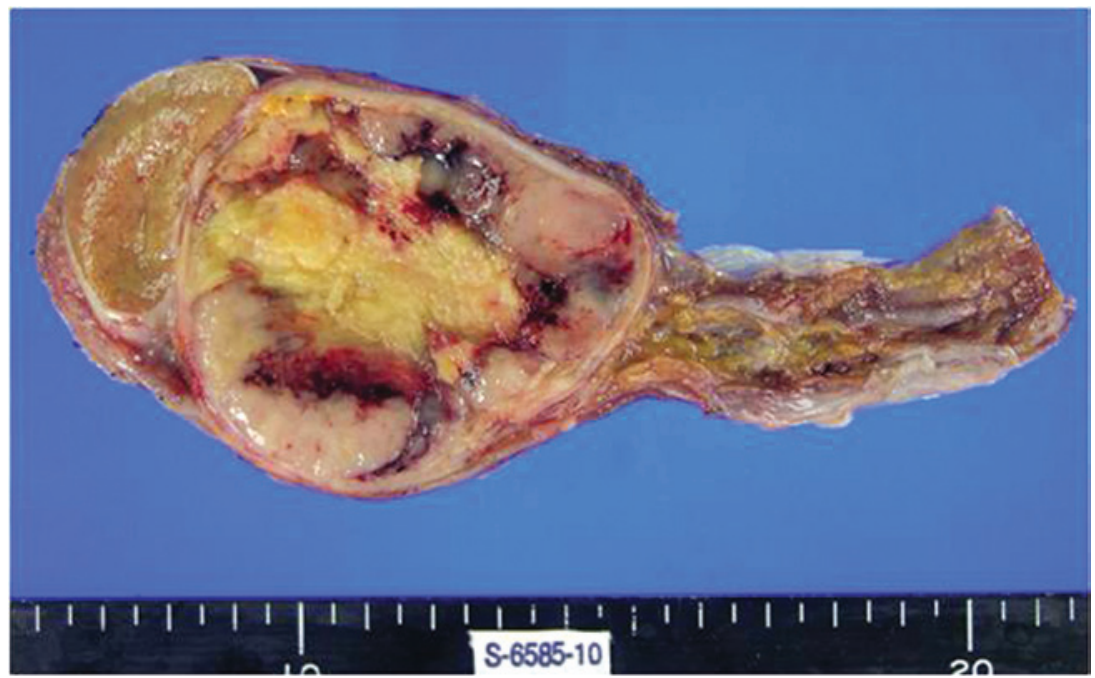

Figure 2. Sectioned surface revealing a well-circumscribed mass with central necrosis and hemorrhage, displacing the testis inferiorly.

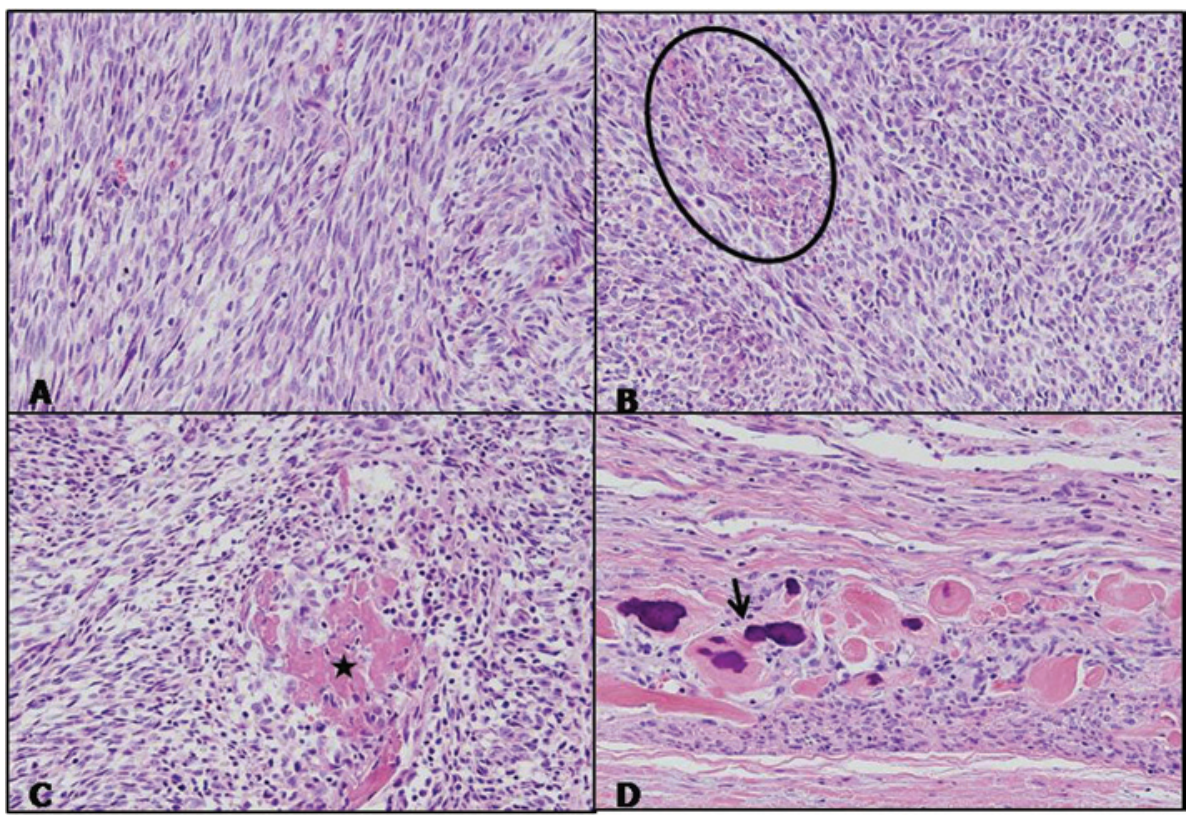

Figure 3. (A and B) Microscopic examination revealed a spindle cell proliferation and focal eosinophilic cytoplasm showing early osteoid formation (circle); (C and D) osteoid matrix (star) and partly mineralized area (arrow). 
pure paratesticular osteosarcoma. Preoperative differential diagnosis appears to be difficult and involves more common intrascral processes, such as inguinal hernia, cord lipoma and testicular mass. Ultrasonograghy (US), computed tomography (CT) scan and magnetic resonance imaging (MRI) may be helpful in defining preoperative diagnosis and the extent of the mass in the neighboring tissue $(9,10)$. In the present case, a pelvic CT scan revealed a large paratesticular mass with calcification and internal necrosis, and suggested sarcoma. When diagnosing extraosseous osteosarcoma, careful clinical and radiological evaluation is mandatory to exclude the possibility of a primary osseous source for the tumor. In the present case, extensive clinical and radiological work-up and thorough histological examination did not reveal a skeletal lesion.

The histogenesis of testicular or paratesticular osteosarcoma is unknown. However, in the case of intratesticular osteosarcoma, two hypotheses have been suggested: i) a neoplastic transformation of sequestered embryonic remains of osteogenic tissue or primitive mesenchymal cells; ii) a transformation of pre-existing teratomatous elements that acquire the potential for a preferential mesenchymal malignancy $(11,12)$.

Optimal local and systemic treatment of paratesticular sarcomas remains controversial, since the information used to guide it is based on small, retrospective series of patients evaluated and treated in a non-uniform manner, and typically spans a number of years. Local relapse is a significant problem following orchiectomy, and reports have suggested that additional local treatment with surgery or irradiation may improve local control (11). In the case of osteosarcoma of paratesticular spermatic cord, complete excision of the tumor with radical inguinal orchiectomy and high ligation of the spermatic cord was the main primary surgical procedure $(1,2,12)$. A more aggressive surgical policy in the management of spermatic cord sarcomas has been proposed as primary surgery, involving wide excision of surrounding soft tissue and re-excision in the case of local recurrence of the disease, since of the patients without clinically apparent disease who underwent re-excision, residual tumor was discovered in almost one third of the cases (13). The prognosis of paratesticular sarcomas is variable from case to case with reports describing early metastasis and multiple recurrences, as well as long-term, disease-free survival. The patient of the first reported osteosarcoma arising in the paratesticular soft tissue succumbed to the disease 6 months following the diagnosis with disseminated disease (4). In the case of primary pure testicular osteosarcoma, inguinal orchi- ectomy with strict and careful follow-up evaluations appears to be a sufficient treatment. However, the prognosis of testicular osteosarcoma is unclear due to the short follow-up duration (14). Lee et al concluded that primary pure testicular osteosarcoma may be associated with a favorable prognosis (16).

In conclusion, we experienced an extremely rare form of osteosarcoma arising directly from the paratesticular soft tissue. This osteosarcoma appears to require a more aggressive treatment strategy compared to testicular osteosarcoma, and may be associated with poor prognosis compared to testicular osteosarcoma.

\section{References}

1. Spirtos G, Abdu RA and Schaub CR: Osteosarcoma of the spermatic cord. J Urol 145: 832-833, 1991.

2. Beiswanger JC, Woodruff RD, Savane PD and Assimos DG: Primary osteosarcoma of the spermatic cord with synchronous bilateral renal cell carcinoma. Urology 49: 957-959, 1997.

3. Stein BS, Petersen RO and Conger KB: Malignant mesenchymoma of the spermatic cord. J Urol 131: 551-552, 1984.

4. Al-Masri A, Al-Shraim M, Abu Al-Samen A, Chetty R and Evans A: Primary paratesticular osteosarcoma: case report and a review of the literature. Sci World J 30; 850-854, 2007.

5. Weiss SW and Goldblum JR: Enzinger and Weiss's Soft Tissue Tumors. 5th edition. Mosby, Inc., Maryland Heights, pp1051-1053, 2008.

6. Sordillo PP, Hajdu SI, Magill GB and Golbey RB: Extraosseous osteogenic sarcoma: a review of 48 patients. Cancer 51: 727, 1983.

7. Klein MJ and Siegal GP: Osteosarcoma: anatomic and histologic variants. Am J Clin Pathol 125: 555, 2006.

8. Russo P, Brady MS, Conlon K, et al: Adult urological sarcoma. J Urol 147: 1032-1036, 1992.

9. Woodward PJ, Schwab C and Sesterhenn IA: Extratesticular scrotal masses: radiologic-pathologic correlation. Radiographics 23: $215-240,2003$

10. Stella M, Di Somma C, Solari N, et al: Primary osteosarcoma of the spermatic cord: case report and literature review. Anticancer Res 27: 1605-1608, 2007.

11. Washecka RM, Mariani AJ,Zuna RE, Honda SA and Chong CDK: Primary intratesticular sarcoma. Immunohistochemical, ultrastructural and DNA flow cytometric study of three cases with a review of the literature. Cancer 44: 1524-1528, 1996.

12. Tazi H, Karmouni T, Ouali M, Koutani A, Hachimi M and Lakrissa A: Osteosarcoma of the testis. Int J Urol 13: 323-324, 2006.

13. Catton C, Jewett M, O'Sullivan B and Kandel R: Paratesticular sarcoma: failure patterns after definitive local therapy. J Urol 161: 1844-1847, 1999.

14. Ballo MT, Zagars GK, Pisters PWT, Feig BW, Patel SR and von Eschenbach AC: Spermatic cord sarcoma: outcome, patterns of failure and management. J Urol 166: 1306-1310, 2001.

15. Coleman J, Brennan MF, Alektiar K and Russo P: Adult spermatic cord sarcomas: management and results. Ann Oncol 38: 635-638, 1999.

16. Lee JS, Choi YD and Choi C: Primary testicular osteosarcoma with hydrocele. Virchows Arch 445: 210-213, 2004. 